\title{
Effective Classical Theory for Real-Time SU $(N)$ Gauge Theories at High Temperature
}

\author{
B. J. Nauta* and Ch. G. van Weert \\ Institute for Theoretical Physics, University of Amsterdam \\ Valckenierstraat 65, 1018 XE Amsterdam, The Netherlands
}

\begin{abstract}
We derive an effective classical theory for real-time $\mathrm{SU}(N)$ gauge theories at high temperature. By separating off and integrating out quantum fluctuations we obtain a $3 \mathrm{D}$ classical path integral over the initial fields and conjugate momenta. The leading hard mode contribution is incorporated in the equations of motion for the classical fields. This yields the gauge invariant hard thermal loop (HTL) effective equation of motion. No gauge-variant terms are generated as in treatments with an intermediate momentum cut-off. Quantum corrections to classical correlation functions can be calculated perturbatively. The 4D renormalizability of the theory ensures that the $4 \mathrm{D}$ counterterms are sufficient to render the theory finite. The HTL contributions of the quantum fluctuations provide the counterterms for the linear divergences in the classical theory.
\end{abstract}

\section{INTRODUCTION}

To understand the processes that have played a role in the early universe, it is important to study the behavior of thermal field theory at high temperatures. Some of the processes with cosmological implications, like sphaleron transitions and the dynamics of weak first-order transitions, are sensitive to the soft modes $\left(k \sim g^{2} T\right.$, with $g$ the gauge coupling) of the magnetic sector in hot gauge theories [1. [2]. This low-momentum behavior is non-perturbative, due to the IR problems of massless field theories in 3D [1, 3].

For static magnetic fluctuations the dominant IR behavior can be isolated from the full quantum theory by the technique of dimensional reduction [4, 5 , which allows one to study the non-perturbative behaviour by standard lattice simulations or other imaginary-time methods [6.7. In a number of papers it has already been argued that real-time thermal field theory in the high- $T$ limit reduces to a classical statistical theory [8] Such a theory consists of equations of motion that govern the time-evolution of the classical fields and an averaging over initial fields with a thermal weight. The goal of this paper is to obtain an effective classical theory by integrating out quantum fluctuations using the method similar to that of dimensional reduction [9].

A major problem for gauge theories is that the perturbative fluctuations (hard modes) do not cleanly decouple from the soft modes one is interested in. Even in lowest order one will have to take the hard-thermal-loop (HTL) effects into account [1,.10]. To handle this in a gauge invariant manner we will propose in the present paper a way of splitting the field in perturbative and non-perturbative modes, other than the standard method of introducing an intermediate scale to separate the soft from the hard modes [10 13]. In this way the influence action obtained by integrating out the hard modes is BRS invariant, in the HTL approximation this implies gauge invariance [14].

the theory that we obtain after the classical approximation is very similar to the effective theory proposed by Iancu [13]. It consists of the HTL equation of motion and a thermal average over initial conditions. The approach that is taken here shows that the HTL's should be calculated with a subtracted propagator. The subtraction follows directly from separating off the path integration over initial fields from the quantum path integral. This subtraction is essential to justify the classical approximation, and it introduces automatically the counterterms for the linear divergences of the classical theory that otherwise have to be introduced by a rather ad hoc matching procedure [13].

The outline of this paper is as follows. In section II we separate off the quantum fluctuations and define an influence action containing the quantum corrections to the classical action. We give the Feynman rules to calculate this action and use it to define the thermal weight and effective equations of motion of the effective classical theory. In section III we calculate the leading order quantum corrections to the thermal weight. We consider especially the terms $\left(\mathcal{E}^{l}\right)^{2}, \mathcal{E}^{l} \mathcal{D}^{l} \mathcal{A}^{0},\left(\mathcal{D}^{l} \mathcal{A}^{0}\right)^{2}$ and $\left(\mathcal{A}^{0}\right)^{2}$, since their coefficients may be different in the Hamiltonian and Lagrangian formulations of static dimensional reduction. The leading order contribution is the Debye mass term, the only static

*E-mail: nauta@wins.uva.nl 
HTL. We then argue that the HTL contributions should be included in the effective equations of motion. The HTL's obtained through integrating out the quantum fluctuations are linearly divergent. These are, in fact, the counterterms for the linear divergences encountered in the effective classical theory. In section IV] we present the effective classical theory and discuss the classical approximation. Section $\mathrm{V}$ contains the conclusion.

\section{SEPARATING OFF THE QUANTUM FLUCTUATIONS}

\section{A. Background fields and zero modes}

In the real-time formulation of thermal field theory, the thermal state is taken into account by extending the time evolution along a contour $C$ in the complex $t$-plane, as shown in fig. 1 . The contour consists of three parts: a forward time branch $C_{1}$ on the real axis starting at an initial time $t_{\text {in }}$ and ending at a final time $t_{\mathrm{f}}$, a backward branch $C_{2}$ from $t_{\mathrm{f}}$ to $t_{\mathrm{in}}$ and a third branch $C_{3}$ down the Euclidean path $t=t_{\text {in }}-i \tau$ from $\tau=0$ to $\tau=\beta$. On this contour we consider a $\mathrm{SU}(N)$ gauge theory for the field $A^{\mu}=\left(A^{0},-\vec{A}\right)$ with generating functional [15, 16],

$$
Z[j]=\int \mathrm{D} A \mathrm{D} E \operatorname{D} \bar{c} \operatorname{D} c \delta(\vec{\nabla} \cdot \vec{A}) \exp \left(i S[A, \vec{E}]+i S_{\mathrm{gh}}[c, \bar{c}, \vec{A}]+i j \cdot A\right)
$$

where $\vec{E}$ is the momentum conjugate to $\vec{A}$. The $\delta$-function enforces the Coulomb gauge $\vec{\nabla} \cdot \vec{A}=0$. We use the inner-product notation $j \cdot A=-2 \operatorname{tr} \int_{C} d^{4} x j_{\mu}(x) A^{\mu}(x)$; the notational conventions are those of reference [16]. The action of the theory is

$$
S[A, \vec{E}]=2 \operatorname{tr} \int_{C} d^{4} x\left[\vec{E} \cdot \partial_{t} \vec{A}+\frac{1}{2}\left(\vec{E}^{2}+\vec{B}^{2}\right)-A^{0} R\right]
$$

Here $\vec{B}$ is the magnetic field and $A^{0}$ is the Lagrange multiplier associated with the constraint $R(A, E):=\vec{\nabla} \cdot \vec{E}+$ $g[\vec{A}, \vec{E}]=0$; it is real on the real-time contour and complex on the imaginary time-branch $A^{0}\left(t_{\text {in }}-i \tau, \vec{x}\right)=i A^{4}(\tau, \vec{x})$. The time-derivative of the field $\vec{A}$ in (2) is taken along the contour. The integration over the ghost action

$$
S_{\mathrm{gh}}=-2 \operatorname{tr} \int_{C} d^{4} x\left(\bar{c} \nabla^{2} c-g \bar{c}[\vec{A}, \vec{\nabla} c]\right)
$$

generates the Faddeev-Popov determinant. The fields $\vec{A}$ and the ghosts are periodic with respect to begin- and endpoint of the contour, but the momenta $\vec{E}$ and the Lagrange multiplier $A^{0}$ have no boundary conditions.

We introduce the classical background fields on the real-time contour $C_{12}=C_{1} \cup C_{2}$ by shifting the fields $A \rightarrow$ $A_{\mathrm{cl}}+A, \vec{E} \rightarrow \vec{E}_{\mathrm{cl}}+\vec{E}$, and similarly for the ghost fields. Performing this shift in the action (2), we obtain

$$
S\left[A_{\mathrm{cl}}+A, \vec{E}_{\mathrm{cl}}+\vec{E}\right]=S[A, \vec{E}]_{\mathrm{cl}}+\delta_{A} S[A, \vec{E}]_{\mathrm{cl}} \cdot A+S\left[A_{\mathrm{cl}}, \vec{E}_{\mathrm{cl}} ; A, \vec{E}\right]
$$

where we have defined $S[A, E]_{\mathrm{cl}}=\left.S[A, E]\right|_{A=A_{\mathrm{cl}}, E=E_{\mathrm{cl}}}$. The last term in (隹) is the part of the shifted action containing terms quadratic-and-higher in the quantum fluctuations. The term linear in $E$ has been made to vanish by imposing on $C_{12}$ the condition $\delta_{E} S[A, E]_{\mathrm{cl}}=0$ which defines the classical electric field in term of the potentials

$$
E_{\mathrm{cl}}^{l}=\partial^{l} A_{\mathrm{cl}}^{0}-\partial^{0} A_{\mathrm{cl}}^{l}-g\left[A_{\mathrm{cl}}^{l}, A_{\mathrm{cl}}^{0}\right]=: F_{\mathrm{cl}}^{l 0}
$$

on $C_{12}$. In section [V], we will require the background field $A_{\mathrm{cl}}$ to satisfy the hard thermal loop (HTL) equations of motion.

The procedure for treating the ghost action (3) is the same as for the gauge field action (4). Linear terms are eliminated by imposing $\delta_{\bar{c}} S_{\mathrm{gh}}[c, \bar{c}, A]_{\mathrm{cl}}=0, \delta_{c} S_{\mathrm{gh}}[c, \bar{c}, A]_{\mathrm{cl}}=0$. Since the ghost fields are not dynamical in the Coulomb gauge, this implies $c_{\mathrm{cl}}=0, \bar{c}_{\mathrm{cl}}=0$.

On the Euclidean part of the contour we follow the prescription of static dimensional reduction and split the fields into zero and non-static modes. The zero modes are constant in time and defined by a projection of the fields on $C_{3}$; for example

$$
\mathcal{A}(\vec{x})=\mathcal{P} A(t, \vec{x})=i T \int_{C_{3}} d t A(t, \vec{x})
$$


The other zero modes are defined similarly and denoted as $\mathcal{E}, \mathcal{C}, \overline{\mathcal{C}}$. The static and the non-static modes are separated in the integration measure by formally writing $D A \rightarrow D \mathcal{A}[D A \delta(\mathcal{P} A-\mathcal{A})]$, and similarly for the other fields. Since in the integration over the quantum fields $A$ the zero mode $\mathcal{A}$ is treated as a constant background field, we may shift $A \rightarrow A+\mathcal{A}$ like on the contour $C_{12}$. The shifted action has the same form as in (4) if we identify $A_{\mathrm{cl}}(x)=\mathcal{A}(\vec{x})$, $E_{\mathrm{cl}}(x)=\mathcal{E}(\vec{x}), C_{\mathrm{cl}}(x)=\mathcal{C}(\vec{x})$ and $\bar{C}_{\mathrm{cl}}(x)=\overline{\mathcal{C}}(\vec{x})$ on $C_{3}$, except that there is no linear term. This is a well known feature of static dimensional reduction. In the present case one may verify that the linear terms vanish on $C_{3}$, because they contain the projections $\mathcal{P} A$, etc.

\section{B. Influence action}

Performing the manipulations described in the previous section, we separate the generating functional (11) into a path integral over static fields

$$
\begin{aligned}
Z[j]= & \int \operatorname{D} \mathcal{A D} \mathcal{E} \operatorname{DC} \operatorname{D\mathcal {C}} \delta(\vec{\nabla} \cdot \overrightarrow{\mathcal{A}}) \\
& \exp \left(i S\left[A_{\mathrm{cl}}, \vec{E}_{\mathrm{cl}}\right]+i S_{\mathrm{gh}}\left[c_{\mathrm{cl}}, \bar{c}_{\mathrm{cl}}, \vec{A}_{\mathrm{cl}}\right]+i W\left[A_{\mathrm{cl}}, \vec{E}_{\mathrm{cl}}, c_{\mathrm{cl}}, \bar{c}_{\mathrm{cl}} ; J\right]+i j \cdot A_{\mathrm{cl}}\right)
\end{aligned}
$$

and one over the quantum fields which defines the influence action

$$
\begin{aligned}
\exp i W\left[A_{\mathrm{cl}}, \vec{E}_{\mathrm{cl}}, c_{\mathrm{cl}}, \bar{c}_{\mathrm{cl}} ; J\right]= & \int \mathrm{D} A_{\mu} \mathrm{D} c \mathrm{D} \bar{c} \delta(\mathcal{P} A) \delta(\mathcal{P} c) \delta(\mathcal{P} \bar{c}) \delta(\vec{\nabla} \cdot \vec{A}) \\
& \exp \left(i S\left[A_{\mathrm{cl}}, \vec{E}_{\mathrm{cl}} ; A\right]+i S_{\mathrm{gh}}\left[c_{\mathrm{cl}}, \bar{c}_{\mathrm{cl}}, A_{\mathrm{cl}} ; c, \bar{c}, A\right]+i J \cdot A\right)
\end{aligned}
$$

Here we used that the gauge constraint may be imposed separately on the zero and non-static modes. The source $J$ is the combination of the external source and the linear term in (4) on $C_{12}$

$$
J_{\mu}=j_{\mu}+\delta_{A^{\mu}} S_{\mathrm{cl}}
$$

The ghost action $S_{\mathrm{gh}}\left[c_{\mathrm{cl}}, \bar{c}_{\mathrm{cl}}, \vec{A}_{\mathrm{cl}} ; c, \bar{c}, \vec{A}\right]$ contains the terms quadratic-and-higher in the quantum fluctuations arising from the shifted ghost action. The Gaussian integration over the momenta $\vec{E}$ has already been performed, which reduces the action in the exponent of (18) to the expression

$$
S\left[A_{\mathrm{cl}}, \vec{E}_{\mathrm{cl}} ; A\right]=-2 \operatorname{tr} \int_{C} d^{4} x\left(-\frac{1}{4} F_{\mu \nu} F^{\mu \nu}+\frac{1}{2} g\left[A^{k}, A^{l}\right] F_{\mathrm{cl}}^{k l}-g A^{0}\left[A^{l}, E_{\mathrm{cl}}^{l}\right]-Q\right),
$$

with the classical field strength $F_{\mathrm{cl}}^{k l}=\partial^{k} A_{\mathrm{cl}}^{l}-\partial^{l} A_{\mathrm{cl}}^{k}-g\left[A_{\mathrm{cl}}^{k}, A_{\mathrm{cl}}^{l}\right], k, l=1,2,3$. The quantum field strength tensor

$$
F^{\mu \nu}=\partial^{\mu} A^{\nu}-\partial^{\nu} A^{\mu}-g\left[A_{c l}^{\mu}, A^{\nu}\right]-g\left[A^{\mu}, A_{c l}^{\nu}\right]-g\left[A^{\mu}, A^{\nu}\right]
$$

contains linear contributions from the background field. The last term in (10) is given by

$$
Q=\frac{1}{2}\left(\mathcal{P} F^{0 l}\right)^{2}
$$

arises from the integration of the momenta and lives only on $C_{3}$.

We note that on the real-time contour $C_{12}$ the action (10) is just the standard quantum action for $\mathrm{SU}(N)$ shifted by a classical background:

$$
S\left[A_{\mathrm{cl}}, \vec{E}_{\mathrm{cl}} ; A\right]+J \cdot A=S\left[A_{\mathrm{cl}}+A\right]-S\left[A_{\mathrm{cl}}\right]+j \cdot A
$$

To derive (13) we inserted the equations of motion (5) for $\vec{E}_{\mathrm{cl}}$ into the action (2), which gives the Lagrangian version $S[A]:=S\left[A, E^{l}=F^{l 0}\right]$ for the $\mathrm{SU}(N)$ action. On $C_{3}$ the electric field $\vec{E}_{\mathrm{cl}}=\overrightarrow{\mathcal{E}}$ is an independent integration variable and we cannot simplify to a form like (13). 


\section{Feynman rules}

The effective action can be calculated perturbatively with the Feynman rules determined by the action (10). By inspection of (13) we see that on $C_{12}$ the interaction vertices are the usual vertices with two, three or four quantum fields on the external lines. Hence, the Feynman rules for a diagrammatic evaluation of vertex functions on $C_{12}$ consist of the usual interactions. But the propagator of the quantum fluctuations is not the usual propagator of thermal field theory, as we will derive below.

On $C_{3}$ there is an interaction containing the static electric field, this interaction gives corrections, for instance, to the $\overrightarrow{\mathcal{E}}^{2}$ term in the classical action. There are also interactions contained in $Q(12)$ these do not contribute at one-loop order and are not considered in this paper. However the quadratic part of $Q$ is however essential in the computation of the propagator of the quantum fluctuations.

The influence action (8) for free fields only, depends on the background fields through the source (9) and will be denoted by $W_{0}[J]$. This quantity determines the propagator $\Delta^{\mu \nu}$ associated with the quantum fluctuations according to

$$
\exp i W_{0}[J]=\exp -\frac{1}{2} \int_{C} d t d t^{\prime} \int \frac{d^{3} k}{(2 \pi)^{3}} \tilde{J}_{\mu}(t,-\vec{k}) \tilde{\Delta}_{C}^{\mu \nu}\left(t-t^{\prime}, \vec{k}\right) \tilde{J}_{\nu}\left(t^{\prime}, \vec{k}\right)
$$

where the tilde indicates the spatial Fourier transform. To evaluate the left-hand-side we insert an integral representation $\delta(\mathcal{P} A)=\int \mathrm{D} \chi_{\mu} \exp (-\chi \cdot A)$ for the delta-function in $(8)$. The auxiliary field $\chi$ is zero everywhere except on $C_{3}$ where it has a spatial dependence: $\chi_{\mu}(t, \vec{x})=\chi_{\mu}(\vec{x}), t \in C_{3}$. The quadratic part of the term $Q$, equation $(12)$, reduces to $Q_{0}=\left(\mathcal{P} \partial_{t} \vec{A}\right)^{2}$, since $\mathcal{P} A^{0}$ vanishes on account of the delta-function constraints in $(\mathbb{8})$. These manipulations allow us to rewrite the free effective action in the form

$$
\exp i W_{0}[J]=\exp \left(\frac{i}{2} \mathcal{P} \partial_{t} \delta_{J} \cdot \mathcal{P} \partial_{t} \delta_{J}\right) \int \mathrm{D}_{C} \chi_{\mu} \exp -\frac{1}{2}(J+i \chi) \cdot D_{C} \cdot(J+i \chi)
$$

with $D_{C}^{\mu \nu}$ the standard thermal propagator of gauge fields in the Coulomb gauge [17]. The contribution of the $Q$ term has been taken out of the functional integration by replacing the spatial gauge fields by the appropriate functional derivatives.

The propagator $\tilde{\Delta}_{C}^{\mu \nu}$ has the same tensor structure as the standard thermal propagator in the Coulomb gauge:

$$
\tilde{\Delta}_{C}^{\mu \nu}\left(t-t^{\prime}, \vec{k}\right)=T^{\mu \nu}(\vec{k}) \tilde{\Delta}_{C}\left(t-t^{\prime}, \vec{k}\right)+g^{\mu 0} g^{\nu 0} \tilde{\Delta}_{C}^{00}\left(t-t^{\prime}, \vec{k}\right)
$$

The matrix $T$ is the transversal projection operator with $T_{0 \nu}=T_{\mu 0}=0$ and

$$
T_{i j}=\delta_{i j}-\frac{k_{i} k_{j}}{k^{2}}
$$

with $k=|\vec{k}|$. The evaluation of the temporal part of the propagator $(16)$ is simple, since the functional derivatives in (15) do not contribute and the thermal propagator $\tilde{D}_{C}^{00}$ is local in time:

$$
\tilde{\Delta}_{C}^{00}\left(t-t^{\prime}, \vec{k}\right)=\tilde{D}_{C}^{00}\left(t-t^{\prime}, \vec{k}\right)-\tilde{S}_{C}^{00}\left(t-t^{\prime}, \vec{k}\right)
$$

with subtraction

$$
\tilde{S}_{C}^{00}\left(t-t^{\prime}, \vec{k}\right)= \begin{cases}0 & t \text { or } t^{\prime} \in C_{12} \\ i \frac{T}{k^{2}} & t, t^{\prime} \in C_{3}\end{cases}
$$

The calculation of the scalar propagator $\tilde{\Delta}_{C}$ for the spatial gauge fields is more involved; we refer to [9] for details. The result is

$$
\tilde{\Delta}_{C}\left(t-t^{\prime}, \vec{k}\right)=\tilde{D}_{C}\left(t-t^{\prime}, \vec{k}\right)-\tilde{S}_{C}\left(t-t^{\prime}, \vec{k}\right),
$$

with $\tilde{D}_{C}$ the standard thermal propagator [17]. The subtraction is given by [9]

$$
\tilde{S}_{C}\left(t-t^{\prime}, \vec{k}\right)= \begin{cases}\frac{T}{k^{2}} \cos k\left(t-t^{\prime}\right) & t, t^{\prime} \in C_{12}, \\ \frac{T}{k^{2}} \cos k\left(t_{\mathrm{in}}-t^{\prime}\right) & t \in C_{3}, t^{\prime} \in C_{12}, \\ \frac{T}{k^{2}} \cos k\left(t-t_{\mathrm{in}}\right) & t \in C_{12}, t^{\prime} \in C_{3}, \\ \frac{T}{k^{2}} & t, t^{\prime} \in C_{3} .\end{cases}
$$


On the real-time part of the contour we recognize the subtraction as the classical propagator. On the vertical part of the contour it is the zero-mode propagator, which implies that static fluctuations on $C_{3}$ do not contribute to the propagator (16). This is what one expects, of course, since static fluctuations are excluded from the path integration in (8).

The propagator for the ghosts can be derived in the same way as for the gauge fields. In the Coulomb gauge it is equal to the propagator for the temporal fluctuations

$$
\tilde{\Delta}_{C}^{\mathrm{gh}}\left(t-t^{\prime}, \vec{k}\right)=\tilde{\Delta}_{C}^{00}\left(t-t^{\prime}, \vec{k}\right) .
$$

The important conclusion is that the IR-limit of the propagator $\tilde{\Delta}_{C}^{i j}$ for the transverse quantum fluctuations is $\mathcal{O}\left(k^{-1}\right)$ and, therefore less singular than the IR-limit $\mathcal{O}\left(k^{-2}\right)$ of the standard thermal propagator $\tilde{D}_{C}^{i j}$. So the severe IR divergences that are present in massless thermal field theories in perturbation theory do not occur in a perturbative evaluation of the influence action. What we have achieved is a clean separation between quantum fluctuations that may be treated perturbatively, and non-perturbative effects contained in the 3D path integral (7).

\section{Thermal weight and equation of motion}

The classical fields are defined on the entire contour $C$. The classical action $S$ and the effective action $W$ in (7), contain contributions from classical fields on the entire contour. The contributions from $C_{3}$ and $C_{12}$ differ completely: the former contributes to the thermal weight whereas the latter determines the time evolution. This difference may be made more explicit by demanding

$$
\vec{A}_{\mathrm{cl}}(t, \vec{x})=\vec{A}_{\mathrm{cl}}(t-i \sigma, \vec{x}), A_{\mathrm{cl}}^{0}(t, \vec{x})=-A_{\mathrm{cl}}^{0}(t-i \sigma, \vec{x}),
$$

with $t \in C_{1}$ and $t-i \sigma \in C_{2}$. Then (5) implies $\vec{E}_{\mathrm{cl}}(t, \vec{x})=-\vec{E}_{\mathrm{cl}}(t-i \sigma, \vec{x})$. We recall that the temporal gauge field and the electric field need not be periodic on the entire contour, so the minus sign in (23) does not cause problems. The consequence is that the contributions to the classical action from the forward and backward time-branch cancel and the contribution from the imaginary time-branch $C_{3}$ remains

$$
i S_{\mathrm{cl}}=-\beta H[\overrightarrow{\mathcal{A}}, \overrightarrow{\mathcal{E}}]+\beta \mathcal{A}^{0} \mathcal{R},
$$

with the abbreviation $\mathcal{A}^{0} \mathcal{R}=-2 \operatorname{tr} \int d^{3} x \mathcal{A}^{0} R[\overrightarrow{\mathcal{A}}, \overrightarrow{\mathcal{E}}]$, and

$$
i S_{\mathrm{gh}}\left[c_{\mathrm{cl}}, \bar{c}_{\mathrm{cl}}, \vec{A}_{\mathrm{cl}}\right]=i S_{\mathrm{gh}}[\mathcal{C}, \overline{\mathcal{C}}, \overrightarrow{\mathcal{A}}]
$$

for the ghost action. The effective action may be split into a part containing only contributions from fields on $C_{3}$ and a part containing contributions from fields both on $C_{12}$ and $C_{3}$

$$
W\left[A_{\mathrm{cl}}, \vec{E}_{\mathrm{cl}}, c_{\mathrm{cl}}, \bar{c}_{\mathrm{cl}}, J\right]=W_{\mathrm{DR}}[\mathcal{A}, \overrightarrow{\mathcal{E}}, \mathcal{C}, \overline{\mathcal{C}}]+W_{\mathrm{RT}}\left[A_{\mathrm{cl}}, \vec{E}_{\mathrm{cl}}, c_{\mathrm{cl}}, \bar{c}_{\mathrm{cl}}, J\right]
$$

with $W_{\mathrm{DR}}$ the influence action of static dimensional reduction and the real-time influence action $W_{\mathrm{RT}}$. The different roles plaid by these two actions becomes clear by noting that $W_{\mathrm{DR}}$ is source independent, while a consequence of (23) is that the real-time action vanishes at zero source $\left.W_{\mathrm{RT}}\right|_{j=0}=0$, as for scalar theories [18]. Hence, the influence action of static dimensional reduction gives the quantum corrections to the thermal weight, while the real-time influence action modifies the source term.

Using the identities (24), (25) and (26) for the generating functional (7), we may write

$$
\begin{gathered}
Z[j]=\int \mathrm{D} \mathcal{A} \operatorname{DE} \operatorname{DC} \operatorname{D} \overline{\mathcal{C}} \delta(\vec{\nabla} \cdot \overrightarrow{\mathcal{A}}) \exp \left(-\beta H[\overrightarrow{\mathcal{A}}, \overrightarrow{\mathcal{E}}]+\beta \mathcal{A}^{0} \mathcal{R}+i S_{\mathrm{gh}}[\mathcal{C}, \overline{\mathcal{C}}, \overrightarrow{\mathcal{A}}]+i W_{\mathrm{DR}}\right) \\
\exp \left(i W_{\mathrm{RT}}+i j \cdot A_{\mathrm{cl}}\right)
\end{gathered}
$$

where the first exponent is the effective thermal weight and the second exponent is the effective source term.

We still have to make a choice for $A_{\mathrm{cl}}$ on the real-time part of the contour. The generating functional is independent of this choice and the idea is to minimize the quantum corrections. This may be implemented by demanding

$$
\left.\delta_{j} W_{\mathrm{RT}}\left[A_{\mathrm{cl}}, \vec{E}_{\mathrm{cl}}, c_{\mathrm{cl}}, \bar{c}_{\mathrm{cl}} ; J\right]\right|_{j=0}=0 .
$$


To rewrite this requirement as an equation of motion for the classical field, we define the effective action

$$
\Gamma\left[A_{\mathrm{cl}}, \tilde{A}\right]=W_{\mathrm{RT}}\left[A_{\mathrm{cl}}, \vec{E}_{\mathrm{cl}}, c_{\mathrm{cl}}, \bar{c}_{\mathrm{cl}} ; J\right]-J \cdot \tilde{A}, \quad \delta_{J} W_{\mathrm{RT}}=\tilde{A}
$$

where $\tilde{A}$ is a general fields on $C_{12}$ (not necessarily equal on $C_{1}$ and $C_{2}$ ). The effective action depends on the zero modes $\mathcal{A}, \overrightarrow{\mathcal{E}}, \mathcal{C}, \overline{\mathcal{C}}$ (not explicitly shown) and the field $\tilde{A}$ on $C_{12}$. Given a source $J$ the field $\tilde{A}$ satisfies

$$
\left.\delta_{A} \Gamma\left[A_{\mathrm{cl}}, A\right]\right|_{A=\tilde{A}}=-J .
$$

Imposing $(29)$ this gives an equation for the classical field on $C_{12}$

$$
\left.\delta_{A} \Gamma\left[A_{\mathrm{cl}}, A\right]\right|_{A=0}=-\left.\delta_{A} S_{12}\right|_{\mathrm{cl}} .
$$

We introduce $\Gamma[A]:=\Gamma[A, \tilde{A}=0]$, where we allow for a general field $A$ on $C_{12}$. Using the identity $\left.\delta_{A} \Gamma\left[A_{\mathrm{cl}}, A\right]\right|_{A=0}=$ $\left.\delta_{A} \Gamma[A]\right|_{\mathrm{cl}}$ (which follows immediately from a generalization of (6.4) in [19]), we may write (31) as

$$
\left.\delta_{A}\left(S_{12}[A]+\Gamma[A]\right)\right|_{\mathrm{cl}}=0 .
$$

The action $\Gamma$ contains the loop corrections to the classical equations of motion. These loop corrections are calculated with the propagator $\Delta$ on the internal lines.

Note that we may set $j=0$ in (28) and (32), since we have initial conditions on the classical fields at a finite time $t_{\text {in }}$ and therefore (32) is not a trivial requirement. This in contrast with situations where one has the asymptotic condition $A_{\mathrm{cl}} \rightarrow 0$, and it is necessary to keep the source in order to obtain non-trivial solutions.

A useful property of the vertex functions defined by $\Gamma[A]$ is that they satisfy the Slavnov-Taylor identities on $C_{12}$. This can be seen by noting that setting the classical fields equal to zero on $C_{12}$ gives the standard action for the gauge fields (13) and the ghost fields. Therefore the action is invariant under BRS transformations of the quantum fields $A$. Also the integration measure $\mathrm{D} A \delta(\mathcal{P} A) \mathrm{D} c \delta(\mathcal{P} c) \mathrm{D} \bar{c} \delta(\mathcal{P} \bar{c})$ is invariant under a BRS transformation on $C_{12}$, since $\mathcal{P}$ does not act on $C_{12}$. From the BRS invariance the Slavnov-Taylor identities follow in a standard manner.

\section{QUANTUM CORRECTIONS}

\section{A. Thermal weight}

In this section we use the previously derived Feynman rules to calculate the leading-order quantum corrections to the thermal weight in the generating functional (27).

Let us assume we may write the effective action as an infinite sum of local terms, symbolically

$$
i W_{\mathrm{DR}}=2 \beta \operatorname{tr} \int d^{3} x \sum K(\partial)^{P}(\mathcal{A})^{Q}(\mathcal{E})^{R}(\mathcal{C})^{S}(\overline{\mathcal{C}})^{S},
$$

where the ghost and anti-ghost fields have the same power since these are Grassmann fields. The factor $\beta$ arises from the imaginary time integration over $C_{3}$. The above equation is a derivative expansion and is valid if the momenta of the fields are small $k<<T$. By power counting and a one-loop estimate we find for the coefficient

$$
K \sim g^{Q+R+2 S} T^{4-N},
$$

with $N=P-Q-2 R-2 S$. We see that terms with $4-N<0$ are suppressed for small momenta $k<<T$ and small fields $g \mathcal{A}, g \mathcal{C}<<T, g \mathcal{E}<<T^{2}$ [5]. Note also that the superficial divergence structure is given by the same power counting: $K$ is superficially finite if $4-N<0$.

We are interested in the leading quantum corrections and we concentrate on terms with $4-N \geq 0$. Since the theory is invariant under static BRS transformations the relevant terms in the effective action must have the form

$$
\begin{aligned}
i W_{\mathrm{DR}}[\mathcal{A}, \overrightarrow{\mathcal{E}}, \mathcal{C}, \overline{\mathcal{C}}]=2 \beta \operatorname{tr} \int d^{3} x & {\left[\frac{1}{4} K_{1} \mathcal{F}^{k l} \mathcal{F}^{k l}+\frac{1}{2} K_{2} \mathcal{E}^{l} \mathcal{E}^{l}-K_{3} \mathcal{E}^{l} \mathcal{D}^{l} \mathcal{A}^{0}+\frac{1}{2} K_{4}\left(\mathcal{D}^{l} \mathcal{A}^{0}\right)\left(\mathcal{D}^{l} \mathcal{A}^{0}\right)\right.} \\
- & \left.\frac{1}{2} m_{0}^{2}\left(\mathcal{A}^{0}\right)^{2}+\frac{1}{4 !} \lambda_{0}\left(\mathcal{A}^{0}\right)^{4}+K_{5}\left(\overline{\mathcal{C}} \partial^{k} \partial^{k} \mathcal{C}-g \overline{\mathcal{C}}\left[A^{k}, \partial^{k} \mathcal{C}\right]\right)+\ldots\right]
\end{aligned}
$$

with $\mathcal{F}^{k l}$ and $\mathcal{D}^{l}$ the static field strength and covariant derivative, respectively. This is the Hamiltonian form of the effective action as found in [20,5]. 
The coefficients $K_{1} \ldots K_{5}, m_{0}^{2}$ and $\lambda_{0}$ in (36) may be calculated in perturbation theory, since the subtractions in (18), (20) and (22) imply that the perturbative expansion is not plagued with the severe IR divergences of thermal field theory.

The mass for the temporal gauge field equals the well known result for the Debye mass

$$
m_{0}^{2}=-2 g^{2} N \mu^{2 \epsilon} \int \frac{d^{d} k}{(2 \pi)^{d}} \frac{d}{d k} \tilde{n}(k)=\frac{1}{3} g^{2} N T^{2},
$$

with $k=|\vec{k}|$. We use dimensional regularization to render the divergent (spatial) momentum integrals finite; $d=3-2 \epsilon$, $\mu$ is a reference mass. The distribution function of the quantum fluctuations is $\tilde{n}(k)=n(k)-T / k$, and comes from the thermal part of the propagator (20). The subtraction $T / k$ introduces a linear divergence in the mass (36). This divergence is temperature dependent in contrast to the $4 \mathrm{D}$ divergences of quantum field theory, and acts as a counterterm for the same divergence arising in the dimensionally reduced theory. In dimensional regularization power divergences are set to zero and the subtraction does not contribute to the mass. Hence the result is the leading order contribution to the Debye mass.

The other constants appearing in (36) are dimensionless $4-N=0$. We find

$$
\begin{aligned}
& K_{2}=K_{3}=-\frac{1}{3} g^{2} N \mu^{2 \epsilon} \int \frac{d^{d} k}{(2 \pi)^{d}} \frac{1}{k^{3}}(1+2 \tilde{n}(k))-\text { c.t. }=-g^{2} N \frac{1}{24 \pi^{2}}\left(\frac{1}{\hat{\epsilon}}+2\right)-\text { c.t. }, \\
& K_{4}=K_{2}+g^{2} N \frac{5}{48 \pi^{2}}\left(\frac{1}{\hat{\epsilon}}+\frac{26}{15}\right)-\text { c.t. },
\end{aligned}
$$

with $\frac{1}{\hat{\epsilon}}=\frac{1}{\epsilon}+\gamma-\log \frac{4 \pi T^{2}}{\mu^{2}}$ and $\gamma$ Eulers constant and c.t. the appropriate counterterms. Note that the subtraction in $\tilde{n}$ avoids a linear infrared divergence in $K_{2}, K_{3}$ and $K_{4}$.

The constants $K_{1}, K_{5}$ and $\lambda_{0}$ have been calculated before in the Lagrangian formulation of dimensional reduction and may be found in [5,20] for covariant gauges. Integrating out the static momenta $\mathcal{E}$ yields the dimensionally reduced action in the Lagrangian formulation.

Unlike for the linear divergence in (36), temperature-independent 4D counterterms are sufficient to render the constants $K_{1}, \ldots, K_{5}, \lambda_{0}$ finite. The constants $K_{1}, \ldots, K_{5}$ are subleading in $g$ compared to the classical action. The term $\lambda_{0}\left(A^{0}\right)^{4}$ is small compared to the term $m_{0}^{2}\left(A^{0}\right)^{2}$ for not too large fields $A^{0} \sim T$, since $\lambda_{0} \sim g^{4}$; see (34). Hence, we conclude that only the $m_{0}^{2}\left(A^{0}\right)^{2}$ is not perturbatively small compared to the classical action. This is the only static HTL and has to be included in the thermal weight for the effective classical theory, while the other subleading contributions can be treated as perturbations.

\section{B. Hard thermal loop contributions to the equation of motion}

We now turn to the equation of motion (32). The effective action $\Gamma$ contains all quantum effects and is a complicated expression. However, since we are interested in low-momentum correlation functions $k<<T$, we may consider only the leading-order terms to the equation of motion at low momenta and weak-coupling.

The leading contributions to $\Gamma$ at low momenta are the well-known HTL's. We review the order estimates [1] 21] for the HTL contributions to the action $\Gamma$ and discuss the modifications due to the subtraction in the quantum propagator.

In the effective classical theory one has two length scales $(g T)^{-1}$ and $\left(g^{2} T\right)^{-1}$. These two scales correspond to two different excitations; the plasmons that are screened over a length scale $(g T)^{-1}[22$, and the attenuating modes 23] that are screened non-perturbatively over a length scale $\left(g^{2} T\right)^{-1}$ due to a magnetic screening mass in $3 \mathrm{D}$ gauge theories [7].

Let us first consider the momentum and energy scale $k \sim g T, k_{0} \sim g T$ of the plasmon modes. We assume that the classical fields are of the same order as the initial fields $A_{\mathrm{cl}} \sim \mathcal{A}$. An estimate for the initial fields is found by requiring that the fields are not suppressed by the thermal weight: $\beta H \sim 1 \rightarrow \mathcal{A} \sim \sqrt{g} T$. This yields the following estimates for terms in the HTL action

$$
\Gamma_{H T L}^{(n)} A_{\mathrm{cl}}^{n} \sim g^{2+\frac{1}{2} n} T^{4}
$$

where $\Gamma_{H T L}^{n}$ are the retarded vertex functions, as they appear in the equations of motion. By definition of the HTL's, the classical and HTL contributions are of the same order. The interaction terms are small compared to the quadratic parts and therefore (resummed) perturbation theory is valid for the plasmon scale $24,25$. 
Secondly we consider the momentum scale $k \sim g^{2} T$ and energy scale $k_{0} \sim g^{4} T$ of the attenuating modes [1]. These are only present in the spatial gauge fields, since the temporal gauge field is electrically screened with Debye screening length $(g T)^{-1}$. Again we assume that the classical fields and initial fields are of the same order, yielding $A_{\mathrm{cl}} \sim \mathcal{A} \sim g T$. We have the following estimates for terms in the classical and HTL action

$$
\Gamma_{H T L}^{(n)} A_{\mathrm{cl}}^{n} \sim g^{2-n} T^{3-n} k_{0} A_{\mathrm{cl}}^{n} \sim g^{6} T^{4} .
$$

Again the HTL contributions are as large as the classical contributions. We see that higher order terms are not small compared to the quadratic part of the action, which means perturbation theory fails. It will not be possible to expand in the non-linearities of the effective classical theory. The estimate for the classical and HTL action (39) is of the order where the perturbation expansion breaks down for the free energy [3].

In thermal field theories large contributions to vertex functions may also come from the IR-region [3]. These may be as large as the HTL and classical contributions. However these contributions are not present in (32) since we use the subtracted propagator, which implies that IR contributions are suppressed by a factor $\beta k \sim g^{2}$. The large IR contributions are included in the effective classical theory. This leads to the conclusion that the HTL's have to be included in the effective equations of motion and that at the scale $g^{2} T$ a perturbation expansion for the effective classical theory fails [10,13].

Let us consider HTL's of the quantum fluctuations. These HTL's are calculated with the Feynman rules given in section II . We focus on the retarded vertex functions, since these appear in the equations of motion. We may restrict ourselves to vertex functions with all (external) times on $C_{12}$, since also correlations between the initial fields and the classical fields at later times can be expressed in these vertex functions as explained in [18]. Since the HTL's consist of one loop diagrams we only need the interactions and quantum propagator on $C_{12}$. The interactions are the usual 3- and 4-point interactions, as we see from (13). The only difference with the usual Feynman rules of thermal field theory is in the propagator, where the Bose-Einstein distribution function is replaced by a subtracted distribution function in the quantum propagator $n_{B E}(k) \rightarrow \tilde{n}(k)=n_{B E}(k)-T / k$. However, we shall now argue that this does not affect the well-know HTL expressions [21] in dimensional regularization. Retarded one-loop diagrams contain one distribution function [21]. The momentum integrals of the usual HTL's have a cut-off at $k \sim T$ due to the Bose-Einstein distribution function yielding a $T^{2}$ behavior. The subtraction in the distribution function of the quantum fluctuations introduces a linear divergence in the HTL's. Since the classical linear divergences arise only at one-loop and the generating functional is finite (including 4D counterterms) the linear divergence in the HTL's acts as a (non-local) counterterm for the classical theory. Since we use dimensional regularization, which sets linear divergences equal to zero, the HTL's of the quantum fluctuations are just the usual HTL's. In appendix A, we analyze the linear divergences using a momentum cut-off on the momenta of the initial fields. It turns out that the linear divergences enter in the plasmon frequency only. Such a linear cut-off dependence has been proposed before from a matching argument 13,26]. The analysis here shows how such a counterterm arises from integrating out the quantum fluctuations.

The HTL's we thus obtain are gauge invariant, just as the usual HTL's. This is as a consequence of BRS invariance of $\Gamma$ and the HTL approximations [14].

\section{EFFECTIVE CLASSICAL STATISTICAL THEORY}

\section{A. Exact generating functional}

In section IID an effective classical statistical theory was defined. Following the literature [1, 10, 13], it was argued that the HTL contributions should be included in the effective Hamiltonian and the equation of motion. We argued also that the HTL's of the quantum fluctuations equal the usual HTL's in dimensional regularization. With this the exact generating functional can be rewritten as

$$
\begin{gathered}
Z[j]=\int \operatorname{D} \mathcal{A} \operatorname{DE} \operatorname{DC} \bar{D} \overline{\mathcal{C}} \delta(\vec{\nabla} \cdot \overrightarrow{\mathcal{A}}) \exp -\beta H_{\mathrm{eff}}[\mathcal{A}, \overrightarrow{\mathcal{E}}]+i S_{\mathrm{gh}}[\mathcal{C}, \overline{\mathcal{C}}, \overrightarrow{\mathcal{A}}]+i W_{\mathrm{DR}}^{\mathrm{cor}} \\
\exp i W_{\mathrm{RT}}+i j \cdot A_{\mathrm{cl}},
\end{gathered}
$$

where we have defined the effective Hamiltonian

$$
H_{\mathrm{eff}}=H-\beta \mathcal{A}^{0} \mathcal{R}+2 \operatorname{tr} \int d^{3} x \frac{1}{2} m_{0}^{2}\left(\mathcal{A}^{0}\right)^{2},
$$

and the static non-HTL corrections 


$$
W_{\mathrm{DR}}^{\mathrm{cor}}=W_{\mathrm{DR}}+2 i \beta \operatorname{tr} \int d^{3} x \frac{1}{2} m_{0}^{2}\left(\mathcal{A}^{0}\right)^{2} .
$$

In appendix B, it is shown this Hamiltonian corresponds to the conserved energy of the classical subsystem.

The HTL equation of motion is

$$
\left[D_{\mathrm{cl}}^{\nu}, F_{\nu \mu}^{\mathrm{cl}}\right](x)=3 \omega_{\mathrm{pl}}^{2} \int \frac{d \Omega}{4 \pi} v_{\mu} \int_{-\infty}^{t} d t^{\prime} U_{\mathrm{cl}}\left(x, x^{\prime}\right) v_{\nu} F_{\mathrm{cl}}^{0 \nu}\left(x^{\prime}\right) e^{-\epsilon\left(t-t^{\prime}\right)}
$$

with $\omega_{\mathrm{pl}}^{2}=g^{2} N T^{2} / 9$ the plasmon frequency. The angular integration is over the direction of $\vec{v},|\vec{v}|=1$. Furthermore, $x^{\prime}=\left(t^{\prime}, \vec{x}-\vec{v}\left(t-t^{\prime}\right)\right)$ and the parallel transporter $U_{\mathrm{cl}}\left(x, x^{\prime}\right)=\mathrm{P} \exp \left(-i g \int_{\gamma} d z_{\mu} A_{\mathrm{cl}}^{\mu}(z)\right)$, with $\gamma$ a straight line from $x$ to $x^{\prime}$. The damping exponential follows from the $\epsilon$-prescription of thermal field theory 17.

The HTL equation of motion has been derived by Blaizot and Iancu [27], using a kinetic approach. They have shown the HTL equation of motion is a linearized Vlasov equation (linear in the deviation from equilibrium), with the r.h.s. of (43) an induced source.

The initial conditions for the equation of motion (43) are

$$
A_{\mathrm{cl}}^{k}\left(t_{\mathrm{in}}, \vec{x}\right)=\mathcal{A}^{k}(\vec{x}) ; F_{\mathrm{cl}}^{0 k}\left(t_{\mathrm{in}}, \vec{x}\right)=\mathcal{E}^{k}(\vec{x}) .
$$

at a finite time $t_{\text {in }}$. The Lagrange multiplier $A^{0}$ is not a dynamical field, so $\mathcal{A}^{0}$ does not act as an initial condition. The path integration in (40) accounts for the thermal average over the initial conditions (44).

\section{B. Classical approximation}

Note that the generating functional $(40)$ is still exact. The necessary approximation to obtain an effective classical theory is to neglect $W_{D R}^{\text {cor }}$ and $W_{R T}$ in (40), which yields

$$
Z_{\mathrm{cl}}[j]=\int \mathrm{D} \mathcal{A} \operatorname{DE} \operatorname{DC} \operatorname{D\mathcal {C}} \delta(\vec{\nabla} \cdot \overrightarrow{\mathcal{A}}) \exp -\beta H_{\mathrm{eff}}[\mathcal{A}, \overrightarrow{\mathcal{E}}]+i S_{\mathrm{gh}}[\mathcal{C}, \overline{\mathcal{C}}, \overrightarrow{\mathcal{A}}]+i j \cdot A_{\mathrm{cl}}
$$

Let us argue why $W_{D R}^{\text {cor }}$ and $W_{R T}$ in (40) may be neglected for the calculation of correlation functions at low momenta. The action $W_{\mathrm{DR}}^{\text {cor }}$ contains only the non-HTL contributions because of (41) and (42). Since these are small compared to the classical and HTL contributions in $H_{\text {eff }}$ the action $W_{\mathrm{DR}}^{\text {cor }}$ may be neglected.

The other approximation $W_{R T}=0$ can be justified as follows. We consider as an example the symmetric transverse two-point function

$$
\tilde{D}_{T}\left(t_{1}-t_{2}, \vec{k}\right)=\frac{1}{4}\left\langle A^{i}\left(t_{1},-\vec{k}\right) T^{i j}(\vec{k}) A^{j}\left(t_{2}, \vec{k}\right)+\left(t_{1} \leftrightarrow t_{2}\right)\right\rangle,
$$

which can be obtained from the generating functional (40)

$$
\tilde{D}_{T}\left(t_{1}-t_{2}, \vec{k}\right)=-\frac{1}{4} T^{i j}\left[\delta_{j_{i}\left(t_{1}-i \sigma\right)} \delta_{j_{j}\left(t_{2}\right)}+\delta_{j_{i}\left(t_{1}\right)} \delta_{j_{j}\left(t_{2}-i \sigma\right)}\right] Z[j],
$$

where the $\sigma$ indicates that the source derivative is taken on the backward contour $C_{2}$, see fig. 1 . Since the classical fields satisfy the effective classical equation of motion the contributions involving $\delta_{j} W_{R T}$ may be neglected. We then find that the two-point function can be separated in a classical and a quantum contribution

$$
\tilde{D}_{T}\left(t_{1}-t_{2}, \vec{k}\right)=\tilde{D}_{T}^{\mathrm{cl}}\left(t_{1}-t_{2}, \vec{k}\right)+\tilde{D}_{T}^{\mathrm{q}}\left(t_{1}-t_{2}, \vec{k}\right)
$$

with

$$
\begin{aligned}
& \tilde{D}_{T}^{\mathrm{cl}\left(t_{1}-t_{2}, \vec{k}\right)}=\frac{1}{2}\left\langle A_{\mathrm{cl}}^{i}\left(t_{1},-\vec{k}\right) T^{i j}(\vec{k}) A_{\mathrm{cl}}^{j}\left(t_{2}, \vec{k}\right)\right\rangle_{\mathrm{cl}}, \\
& \tilde{D}_{T}^{\mathrm{q}}\left(t_{1}-t_{2}, \vec{k}\right)=-\frac{1}{4}\left\langle\left[\delta_{j\left(t_{1}-i \sigma\right)} \delta_{j\left(t_{2}\right)}+\delta_{j\left(t_{1}\right)} \delta_{j\left(t_{2}-i \sigma\right)}\right] W_{R T}\right\rangle_{\mathrm{cl}} .
\end{aligned}
$$

The brackets $\langle\ldots\rangle_{\mathrm{cl}}$ denote the classical average over the initial conditions defined by (45). In temporal Fourier space these contributions take the form 


$$
\begin{aligned}
& \tilde{D}_{T}^{\mathrm{cl}}\left(k_{0}, \vec{k}\right)=\rho^{\mathrm{cl}}\left(k_{0}, \vec{k}\right) n_{\mathrm{cl}}\left(k_{0}\right), \\
& \tilde{D}_{T}^{\mathrm{q}}\left(k_{0}, \vec{k}\right)=\rho^{\mathrm{q}}\left(k_{0}, \vec{k}\right) \frac{1}{2}\left[1+2 \tilde{n}\left(k_{0}\right)\right],
\end{aligned}
$$

with the classical spectral density

$$
\rho^{\mathrm{cl}}\left(k_{0}, \vec{k}\right)=2 \operatorname{Im}\left[\frac{1}{-\left(k_{0}+i \epsilon\right)^{2}+\vec{k}^{2}+\Pi_{R}^{\mathrm{cl}}\left(k_{0}, \vec{k}\right)}\right],
$$

and $\Pi_{R}^{\mathrm{cl}}\left(k_{0}, \vec{k}\right)$ the classical retarded self-energy. A similar expression can be given for the spectral density $\rho^{\mathrm{q}}$ with self-energy $\Pi_{R}^{\mathrm{q}}$. The appearance of $\tilde{n}=n_{B E}-T / k_{0}$ in (52), instead of the Bose-Einstein distribution function, is a consequence of the subtraction in the free propagator (20). In fact, in the free case $\left(\Pi_{R}^{\mathrm{q}}=0\right)$ the two-point function (52) corresponds to the free propagator $(20)$. Note that in the case that the classical fields satisfy the effective equation of motion (32) exactly $\delta_{j} W_{R T}$ vanishes identically and as a consequence the self-energies $\Pi_{R}^{\mathrm{cl}}, \Pi_{R}^{\mathrm{q}}$ are equal to the full self-energy. We have required that the classical fields satisfy the HTL equation of motion, in this case the leading contribution to both the self-energies $\Pi_{R}^{\mathrm{cl}}, \Pi_{R}^{\mathrm{q}}$ is the HTL self-energy. Indeed, the action $W_{R T}$ contains the HTL self-energy and all other HTL vertices. However the quantum contribution $D_{T}^{\mathrm{q}}$ to the symmetric transverse two-point function can be neglected at small momenta $\left|k_{0}\right|<<T$, since

$$
\frac{1}{2}\left[1+2 \tilde{n}\left(k_{0}\right)\right]=\frac{k_{0}}{12 T}+\mathcal{O}\left(k_{0}^{2} / T^{2}\right)<<\frac{T}{k_{0}}, \quad 0<k_{0}<<T .
$$

Therefore we may neglect the action $W_{R T}$ for the calculation of (symmetric) correlation functions at low momenta. Note that the subtraction in the two-point function for the quantum fluctuations is essential to justify the classical approximation (45) for the calculation of low-momenta correlation functions.

\section{CONCLUSION}

We have derived an effective classical theory by integrating out quantum fluctuations. The effective equation of motion (32) is is BRS invariant, which in the HTL approximation implies gauge invariance. The Feynman rules for the calculation of the effective equation of motion are the same as usual except for a subtraction in the propagator. At real-times (on the contour $C_{12}$ ) this subtraction enters only in the distribution function $\tilde{n}=n_{B E}-T / k$. We would like to stress that this subtraction is a direct consequence of the extraction of the path integration over initial fields from the quantum path integral. This subtraction is essential in two respects. It allows one to argue that the corrections from $W_{R T}$ to symmetric correlation functions are small and therefore the classical approximation is justified. Secondly, the subtraction introduces linear divergences in the HTL equations of motion and the effective Hamiltonian that act as counterterms for the linear divergences in the classical theory. We used dimensional regularization which sets linear divergences equal to zero. Since a non-perturbative treatment of the classical theory requires a lattice implementation, the generalization to a lattice regularization is important, see [28].

There are still logarithmic divergences present in the effective classical theory (45). Therefore the classical theory (45) has to be formulated with some cut-off $\Lambda$, which has to be large compared to the plasmon scale $g T<<\Lambda$. Since logarithmic divergences are suppressed by a factor $k / T \sim g, g^{2}$ (at one-loop) or $g^{2}$ (at two-loops) the cut-off may be taken as large as the temperature $\Lambda \sim T$, since then the requirement $(k / T) \log (\Lambda / g T), g^{2} \log (\Lambda / g T)<<1$ is fulfilled.

In principle, $\Gamma$ and $W_{\mathrm{DR}}$ furnish the logarithmic counterterms. If these can be extracted explicitly the result is a cut-off independent classical approximation. The counterterms for the one-loop divergences of the classical theory may be extracted from the one-loop logarithmic divergent contributions in $\Gamma$ and $W_{\mathrm{DR}}$. However, it is unclear how one obtains the counterterms for the two-loop logarithmic divergences. Yet, we know that the full one-loop contributions together with the two-loop logarithmic divergent contributions should be sufficient. This suggests that an analysis as in 29] for static dimensional reduction may be generalized successfully.

\section{ACKNOWLEDGMENTS}

We would like to thank G. Aarts and J. Smit for useful discussions. 


\section{APPENDIX A: HARD THERMAL LOOPS WITH A SHARP CUT-OFF}

To explicitly see the linear divergence in the HTL's, we use a sharp cut-off $\Lambda$ on the momenta of the classical fields at $t_{\text {in }}$ to regularize divergent momentum integrals. This imposes the restriction $k<\Lambda$ on the initial fields and consequently on the subtraction (21) on $C_{12}$. Repeating the arguments of section IIIB, we see that this reduces to a replacement of the Bose-Einstein distribution function of the usual HTL's by $\tilde{n}_{\Lambda}$

$$
n(k) \rightarrow \tilde{n}_{\Lambda}(k)=n(k)-\frac{T}{k} \Theta(\Lambda-k)
$$

In the HTL expressions for the retarded vertex functions, we use the expansion

$$
\int d k k^{2} \tilde{n}_{\Lambda}(k+p \cos \Theta)=\int d k\left[k^{2} \tilde{n}_{\Lambda}(k)-2 k \tilde{n}_{\Lambda}(k) p \cos \Theta+\ldots\right]
$$

in the external momenta $p$. The angular integration decouples from the $k$ integration, as usual in the HTL approximation. Performing the $k$ integration we find the familiar HTL contributions to the vertex functions, but with a $\Lambda$-dependent plasmon frequency

$$
\left(\omega_{\mathrm{pl}}^{\Lambda}\right)^{2}=\omega_{\mathrm{pl}}^{2}-\frac{2}{3 \pi^{2}} g^{2} N \Lambda T
$$

This leads to the conclusion that a linear divergence appears in the plasmon frequency. By the same reasoning as in section III B, it is clear that this linear divergence acts as a counterterm for the classical theory.

In the literature [10,30,31, often a cut-off is introduced according to

$$
n(k) \rightarrow n_{\Lambda}(k)=n(k) \Theta(\Lambda-k) .
$$

In the classical approximation, one chooses $g T<<\Lambda<<T$ and approximates the soft field by a classical field [10,30. The classical theory then contains the leading order contributions to low-momentum correlation functions up to corrections of order $\beta \Lambda$. However, the result including all contributions from integrating out the hard modes is not exact anymore. The reason is that an approximation is made on the low-momentum field, namely that it behaves classically. This implies that the dependence on the cut-off does not cancel out in the final result. A linear dependence on the cut-off will remain.

\section{APPENDIX B: EFFECTIVE HAMILTONIAN}

In this appendix we consider the classical subsystem, that is the generating (40) functional without the quantum corrections $W_{\mathrm{DR}}^{\text {cor }}=0=W_{\mathrm{RT}}$. We show that the effective Hamiltonian in (41) corresponds to the energy of the classical subsystem. We follow Blazoit and Iancu [27] and introduce an auxiliary field $W^{0}(x, v)$, that is also a function of the direction of $\vec{v} ; v=(1, \vec{v})$ and $|\vec{v}|=1$. This allows for a local formulation of the equations of motion (43)

$$
\begin{gathered}
{\left[D_{\mathrm{cl}}^{\nu}, F_{\nu \mu}^{\mathrm{cl}}\right](x)=3 \omega_{\mathrm{pl}}^{2} \int \frac{d \Omega}{4 \pi} v_{\mu} W^{0}(x, v)} \\
{\left[v_{\nu} D_{\mathrm{cl}}^{\nu}, W^{0}(x, v)\right]=F_{\mathrm{cl}}^{0 \rho}(x) v_{\rho},}
\end{gathered}
$$

with the covariant derivative $D_{\mathrm{cl}}^{\mu}=\partial^{\mu}+i g A_{\mathrm{cl}}^{\mu}$. The conserved energy of this system (B1, B2) is 27

$$
E=-\operatorname{tr} \int d^{3} x\left[\vec{E}^{2}(x)+\vec{B}^{2}(x)+3 \omega_{\mathrm{pl}}^{2} \int \frac{d \Omega}{4 \pi} W^{0}(x, v) W^{0}(x, v)\right] .
$$

In particular, at $t_{\text {in }}$ the energy is

$$
E=-\operatorname{tr} \int d^{3} x\left[\overrightarrow{\mathcal{E}}^{2}(\vec{x})+\overrightarrow{\mathcal{B}}^{2}(\vec{x})+3 \omega_{\mathrm{pl}}^{2} \int \frac{d \Omega}{4 \pi} W^{0}\left(t_{\mathrm{in}}, \vec{x}, v\right) W^{0}\left(t_{\mathrm{in}}, \vec{x}, v\right)\right] .
$$

The value of the auxilary field at $t_{\text {in }}$ follows from the prescription to keep the fields constant in time before $t_{\text {in }}$ in (43). In a time-independent background field the vector current is zero, hence, the vector current at $t_{\text {in }}$ vanishes. It follows that $W^{0}\left(t_{\mathrm{in}}, \vec{x}, v\right)$ is independent of the direction of $\vec{v}$. A calculation yields 


$$
W^{0}\left(t_{\text {in }}, \vec{x}, v\right)=A^{0}\left(t_{\text {in }}, \vec{x}\right) .
$$

Using the equation of motion at $t_{\text {in }}$, we have

$$
\left[D_{\mathrm{cl}}^{l}, F_{l 0}^{\mathrm{cl}}\right]\left(t_{\mathrm{in}}, \vec{x}\right)=\mathcal{R}(\vec{x})=3 \omega_{\mathrm{pl}}^{2} A^{0}\left(t_{\mathrm{in}}, \vec{x}\right) .
$$

Inserting ( $\mathrm{B} 5$ ) and $(\mathrm{B} 6)$ in ( $\mathrm{B} 4)$, we find for the energy of the classical subsystem

$$
E=-\operatorname{tr} \int d^{3} x\left[\overrightarrow{\mathcal{E}}^{2}(\vec{x})+\overrightarrow{\mathcal{B}}^{2}(\vec{x})+\frac{1}{3 \omega_{\mathrm{pl}}^{2}} \mathcal{R}^{2}(\vec{x})\right] .
$$

Let us consider the effective Hamiltonian (41). Since we neglect the influence actions in (40), we can simply integrate out the zero mode of the temporal gauge field $\mathcal{A}^{0}$. This yields the effective Hamiltonian $H_{\text {eff }}=E$ in the thermal weight.

An effective classical theory based on the equations of motion (B1, B2 $)$, has been proposed by Iancu [13]. The average over the initial conditions is taken by a path integration over $\overrightarrow{\mathcal{A}}, \overrightarrow{\mathcal{E}}$ and $W^{0}\left(t_{\mathrm{in}}, \vec{x}, v\right)$, constrained by Gauss' law. We see from (B5), that restricting the path integration over $\vec{v}$-independent auxiliary fields at $t_{\text {in }}$, is consistent with the inclusion of the correlations between initial fields and the fields at later times according to the prescription in (43). The value for initial auxiliary field is then determined by Gauss' law (B6). This yields a somewhat simpler effective classical theory than proposed by Iancu, since no path integration over $W^{0}\left(t_{\text {in }}, \vec{x}, v\right)$ has to be performed.

[1] P. Arnold, D. Son and L.G. Yaffe, Phys. Rev. D55 (1997) 6264.

[2] G.D. Moore, in proceedings of the conference Strong and Electroweak matter '97, Eger, Hungary, p. 108.

[3] A.D. Linde, Phys. Lett. B96 (1980) 289.

[4] E. Braaten, Phys. Rev. Lett. 74 (1995) 2164.

[5] K. Kajantie, M. Laine, K. Rummukainen, M. Shaposhnikov, Nucl. Phys. B458 (1996) 90.

[6] K. Kajantie, M. Laine, K. Rummukainen and M. Shaposhnikov, Nucl. Phys. B503 (1997) 357;

K. Kajantie, M. Laine, A. Rajantie, K. Rummukainen and M. Tsypin, JHEP 9811 (1998) 011;

F. Karsch, M. Oevers and P. Petreczky, Phys. Lett. B442 (1998) 291;

M. Guertler, E.-M. Ilgenfritz, J. Kripfganz, H. Perlt and A. Schiller, Nucl. Phys. B483 (1997) 383.

[7] W. Buchmüller, O. Philipsen, Nucl. Phys. B443 (1995) 47;

G. Alexanian, V.P. Nair, Phys. Lett. B352 (1995) 435;

F. Eberlein, Phys. Lett. B439 (1998) 130.

[8] G. Aarts and J. Smit, Phys. Lett. B393 (1997) 395; Nucl. Phys. B511 (1998) 451;

W. Buchmüller and A. Jakovác, Phys. Lett. B407 (1997) 39.

[9] B.J. Nauta and Ch.G. van Weert, Phys. Lett. B444 (1998) 463.

[10] D. Bödeker, L. McLerran and A. Smilga, Phys. Rev. D52 (1995) 4675.

[11] D. Son, hep-ph/9707351.

[12] C. Hu and B. Muller, Phys. Lett. B409 (1997) 377;

G. D. Moore, C. Hu and B. Muller, Phys. Rev. D58 (1998) 045001.

[13] E. Iancu, Phys. Lett. 435 (1998) 152.

[14] R. Kobes, G. Kunstatter and A. Rebhan, Nucl. Phys. B355 (1991), 1.

[15] L.D. Faddeev and A.A. Slavnov, Gauge Fields: Introduction to Quantum Theory (Addison-Wesley Publishing Company, Redwood City, 1991) p. 1.

[16] C. Itzykson and J.-B. Zuber, Quantum field theory (McGraw-Hill, New York, 1980) p. 1.

[17] M. Le Bellac, Thermal Field Theory (Cambridge University Press, Cambridge, 1996) p. 1.

[18] B.J. Nauta and Ch.G. van Weert, in preparation.

[19] Y. Fujimoto, L.O. Raifeartaigh and G. Parravicini, Nucl. Phys. B212 (1983) 268.

[20] N.P. Landsman, Nucl. Phys. B322 (1989) 498.

[21] E. Braaten and R. D. Pisarski, Nucl. Phys. B337 (1990) 569.

[22] V.V. Klimov, Sov. J. Nucl. Phys. 33 (1981) 934.

H.A. Weldon, Phys. Rev. D26 (1982) 1394.

[23] V.V. Lebedev and A.V. Smilga, Ann. Phys. 202 (1990) 229.

[24] E. Braaten and R. D. Pisarski, Phys. Rev. Lett. 64, 1338.

[25] H. Schulz, Nucl. Phys. B413, 353. 
[26] G. Aarts, in Proceedings of the Conference Strong and Electroweak Matter '97, Eger, 1997, edited by F. Csikor and Z. Fodor.

[27] J.P. Blaizot and E. Iancu, Nucl. Phys. B417 (1994) 608; Nucl. Phys. B421 (1994) 565.

[28] B.J. Nauta, hep-ph/9906389.

[29] A. Jakovác, Phys. Rev. D53 (1996) 4538.

[30] W. Buchmüller and A. Jakovác, Nucl. Phys. B521 (1998) 219; A. Jakovác, Phys. Lett. B446 (1999) 203.

[31] M. D'Attanasio and M. Pietroni, Nucl. Phys. B498 (1997) 443. 


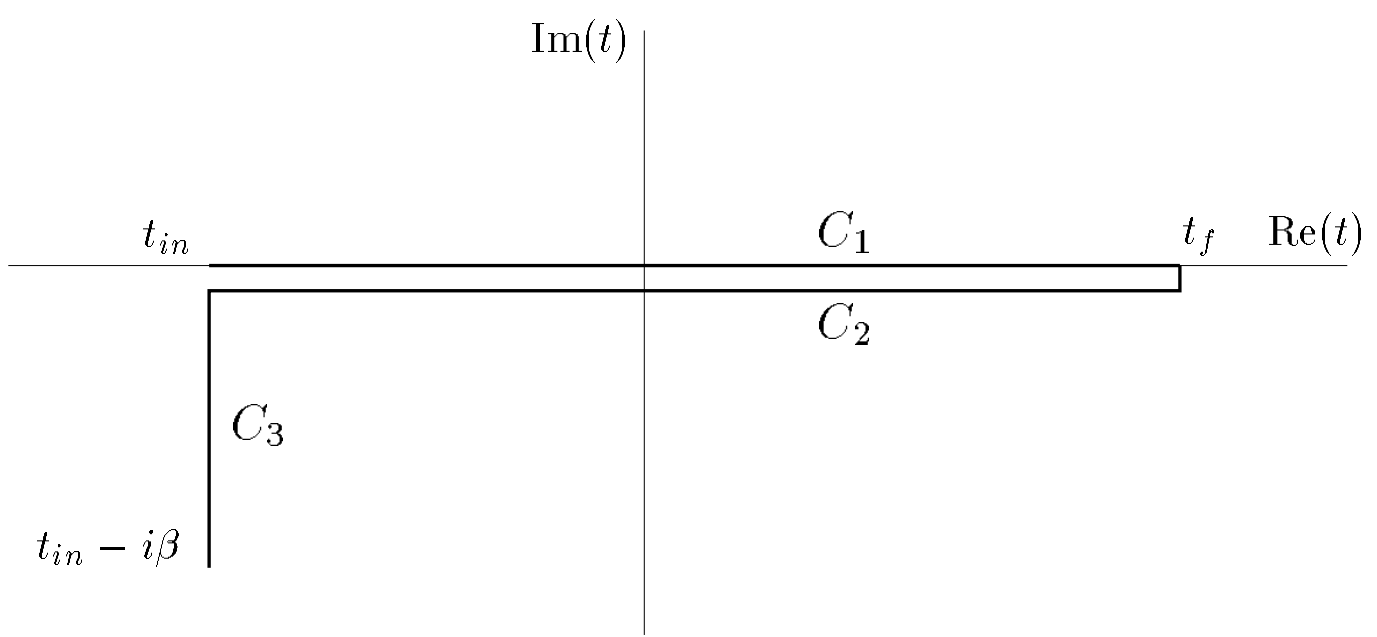

FIG. 1. The time contour $C$. 